\title{
Conventional Nanoindentation in Self-Assembled Monolayers Deposited on Gold and Silver Substrates
}

\author{
Leila Costelle, ${ }^{1,2}$ Liina Lind, ${ }^{3,4}$ Pasi Jalkanen, ${ }^{1,3}$ Minna T. Räisänen, ${ }^{5}$ \\ Roman Nowak, ${ }^{3}$ and Jyrki Räisänen ${ }^{1}$ \\ ${ }^{1}$ Department of Physics, University of Helsinki, Box 64, 00014 Helsinki, Finland \\ ${ }^{2}$ Surface Science Laboratory, Optoelectronics Research Centre, Tampere University of Technology, P.O. Box 692, 33101 Tampere, Finland \\ ${ }^{3}$ Nordic Hysitron Laboratory, Department of Materials Science and Engineering, Aalto University, P.O. Box 16200, 00076 Aalto, Finland \\ ${ }^{4}$ Department of Materials Engineering, Tallinn University of Technology, Ehitajate tee 5, 19086 Tallinn, Estonia \\ ${ }^{5}$ Department of Chemistry, University of Helsinki, P.O. Box 55, 00014 Helsinki, Finland
}

Correspondence should be addressed to Jyrki Räisänen, jyrki.raisanen@helsinki.fi

Received 4 April 2012; Revised 15 June 2012; Accepted 16 June 2012

Academic Editor: Zhi Li Xiao

Copyright () 2012 Leila Costelle et al. This is an open access article distributed under the Creative Commons Attribution License, which permits unrestricted use, distribution, and reproduction in any medium, provided the original work is properly cited.

\begin{abstract}
Self-assembled monolayers (SAMs) are promising materials for micromechanical applications. However, characterization of mechanical properties of monolayers is challenging for standard nanoindentation, and new efficient analysis techniques are needed. Hereby, a conventional nanoindentation method has been combined in a unique way with efficient data analysis based on consumed energy calculation and load-displacement data. The procedure has been applied on SAMs of 4,4'-biphenyldithiol (BPDT) on Au, 1-tetradecanethiol (TDT), and 1-hexadecanethiol (HDT) on Au and Ag substrates being the first study where SAMs of the same thiols on different substrates are analyzed by nanoindentation providing a new insight into the substrate effects. Unlike TDT and HDT SAMs, which are found to strongly enhance the homogeneity and stiffness of the underlying substrate, the BPDT covered Au substrate appears softer in mechanical response. In the case of TDT and HDT SAMs on Ag the structures are softer showing also faster relaxation than the corresponding structures on Au substrate. The proposed procedure enables a fast and efficient way of assessing the complex behaviour of SAM modified substrates. As a consequence, the results are relevant to practical issues dependent on layer activity and toughness.
\end{abstract}

\section{Introduction}

Self-assembled monolayers (SAMs) have already found applications in chemistry, biology and physics $[1,2]$. For example, a hydrophobic monolayer of 1-hexadecanethiol (HDT) has a low friction coefficient [1] and therefore may be used as a solid lubricant in a MEMS device [3]. However, it is necessary to take into account that the volume of modern MEMS devices is very small. Therefore, distances between the surfaces of components achieve their absolute limits, making physical contacts between materials more likely [3].

From applications point of view, it is crucial to know the mechanical properties of SAMs [1, 2, 4-12]. Furthermore, statistical evaluation of the experimental data is increasingly needed in mechanical testing as size effects and statistical variation of data are getting more prominent with decreasing dimensions [13]. For instance, the plastic behaviour of metals can be predicted from the statistical averages of separated defect nucleation and migration events [14]. Therefore standardized experimental procedures and analysis methods describing the statistical properties of the reference materials in nanoscale are needed for quantitative analysis of more complicated systems, such as SAMs. In the literature, mechanical properties of alkanethiol/biphenyl thiol SAMs on Au substrate have been determined experimentally by employing a sharp or spherical indenter (radius 20-200 nm) which has been pressed onto the sample surface using relatively high loads (in the $\mu \mathrm{N}$ range). Consequently, the indentation depths have varied between 10 and $190 \mathrm{~nm} \mathrm{[4,}$ $7,8,11]$. Also a few molecular dynamics simulation studies have been performed for systems consisting of alkanethiol SAMs on Au. In these cases, the indentation depths have been very shallow being only of $0.5-1.5 \mathrm{~nm}[5,6,12]$. 
We have previously studied mechanical responses of 1-dodecanethiol and 11-mercaptoundecanoic acid SAMs, which are of same height but different hydrophobicity, using ultralow load contact probing with a relatively large spherical diamond tip [15]. Here we go well beyond these initial results and present the indentation response of HDT and 1-tetradecanethiol (TDT) SAMs which both have a- $\mathrm{CH}_{3}$ terminal group but height difference of two $-\mathrm{CH}_{2}-$ units, and for 4,4'-biphenyldithiol (BPDT) which forms a less ordered SAM than alkanethiols and has potential stronger interaction with the indenter tip through free $-\mathrm{SH}$ groups. The aim for using a relatively large spherical tip (radius of $\sim 2 \mu \mathrm{m}$ ) is to probe a greater area of the monolayer in order to avoid the effect of local structural variations within the SAM layer. Indeed, the local variations of the van der Waals bonds between the molecules would not affect drastically the nanoindentation results, buckling of the molecules at the surface asperities is minimized, and the better statistics is obtained for larger indenter-layer contact area.

The similar idea (usage of virtually flat punch indenter to increase the contact area) was already advocated for MDsimulations of indentation in crystalline clusters to display average response of a crystal $[13,16]$. However, it proved for the first time of practical importance through our previous [15] and present publications.

SAM layers thickness ranges usually from 2 to $4 \mathrm{~nm}$ and depends on the actual length of the molecules and their orientation on the substrate [5]. The size of the molecules studied in the present study are about $1.60 \mathrm{~nm}$ (TDT), $1.70 \mathrm{~nm}$ (HDT), and $1.25 \mathrm{~nm}$ (BPDT) [17-19], which require ultra shallow indentation depth (beyond all the limits recognized for the indentation technique). Hence, our demonstration that different monolayers are showing dissimilar response already at $2 \mathrm{~nm}$ indentation depth range is of great importance to application of this particular method. We have to confess that the consistent, repeatable results we obtained employing the hitherto conventional indentation method to requiring single-molecular SAM films appeared to us as a pleasant surprise that surpasses all expectations. Indeed we confirmed that nanoindentation in its conventional form can provide sound information on the mechanical behaviour of SAMs on various metallic substrates and to properly resolve the substrate effect.

\section{Experimental}

2.1. Materials and Sample Preparation. For the preparation of the SAMs, a well-established and generally approved preparation procedure was used (for details refer to [20]). The Au and Ag substrates were prepared by thermal evaporation of $\sim 150 \mathrm{~nm}$ of $\mathrm{Au}$ and $\mathrm{Ag}$ on $\mathrm{Si}(100)$. The Au specimens were primed with a $\sim 5 \mathrm{~nm}$ thick Ti layer for better adhesion. The (111) orientation of the evaporated $\mathrm{Au}$ and Ag films on Si wafers was confirmed with X-ray diffraction analysis. Selfassembled monolayers of TDT $\left(\mathrm{CH}_{3}-\left(\mathrm{CH}_{2}\right)_{13}-\mathrm{SH}\right)$ and HDT $\left(\mathrm{CH}_{3}-\left(\mathrm{CH}_{2}\right)_{15}-\mathrm{SH}\right)$, terminated with hydrophobic$\mathrm{CH}_{3}$ tail, as well as BPDT (HS- $\left.\mathrm{C}_{6} \mathrm{H}_{4}-\mathrm{C}_{6} \mathrm{H}_{4}-\mathrm{SH}\right)$, terminated with hydrophilic-SH tail, were formed by immersing freshly evaporated gold and silver substrates in $1 \mathrm{mM}$ EtOH (absolute) solution of the respective molecules for $24 \mathrm{~h}$ at room temperature. After removal from the solutions, the samples were rinsed with $\mathrm{EtOH}$ and dried in a nitrogen stream. All thiols were purchased from Aldrich and used as received.

2.2. Nanoindentation. The nanoindentation experiments as well as the surface topography evaluation were conducted using the commercially available nanoindentation equipment (Hysitron Triboindenter-, Hysitron Inc., Minneapolis, $\mathrm{MN}$ ) enabling us probe the mechanical response of the investigated layers as well as to image the surface features in low-load contact mode. The stated transducer noise floor levels of loading and depth scale were as low as $\pm 50 \mathrm{nN}$ and $\pm 0.1 \mathrm{~nm}$, respectively. The effect of the instrumental drift was found negligible as it is compensated for in the indentation procedure. The total time of the indentation cycle is kept short compared to nominal drift rate (shorter than $0.02 \mathrm{~nm} / \mathrm{s}$ ). The same spherical diamond indenter with the nominal tip radius $R$ of $2 \mu \mathrm{m}$ was used for surface imaging and profiling as well as loading.

The indented samples were in a dry state and they were subjected to nanoindentation cycles consisting of 5 seconds loading and unloading with 2 seconds dwell time at the peak load of $10 \mu \mathrm{N}$, which resulted in average loading/unloading rate of $2 \mu \mathrm{N} / \mathrm{s}$. The indentations were performed along the a priori programmed pattern that consisted of 5 to 25 points with $5 \mu \mathrm{m}$ lateral and horizontal separation between indentation marks. A total of 25 to 125 indentation tests were performed on each of the specimens that to our experience secured reliability of the obtained results.

Furthermore, having in mind the critical experimental limitations that apply to requiring SAM samples, the special experimental precautions have been applied to secure high accuracy of the indentation probing. They include: rigorous stabilization of temperature inside the indenter chamber (variation less than $0.05 \mathrm{deg}$ ), humidity kept at a constant level, and placement of specimens in a chamber several hours before the test to allow the stabilization of the environment.

In closing, one may recognize the scale of our experiment using a simple evaluation. Since the approximate contact area of a tip (at the indentation depth of $1 \mathrm{~nm}$ ) equalled $12500 \mathrm{~nm}^{2}$, and the cross-section of SAM molecule is close to $2.1 \mathrm{~nm}^{2}$ [21], our probing involved over 5000 molecules with an average load per molecule of $2 \mathrm{nN}$ (at peak-load). The above rough estimation gives a reader quite instructive picture of the probing we carried out.

\section{Results and Discussion}

Nanoindentation experiments were performed for five different SAM/substrate systems (TDT/Au, HDT/Au, BPDT/Au, TDT/Ag and HDT/Ag) and for pristine Au and Ag substrates. For data analysis, we measured the average maximum indentation depth before the dwell time together with the standard deviation at $9.5 \mu \mathrm{N}$ and calculated the consumed energy over the loading-unloading cycle for each sample [15]. The obtained data is summarised in Table 1. 
TABLE 1: Statistical data obtained for pristine Au and Ag substrates and SAM/substrate systems.

\begin{tabular}{lccc}
\hline & Average maximum depth at $9.5 \mu \mathrm{N}(\mathrm{nm})$ & Standard deviation at $9.5 \mu \mathrm{N}(\mathrm{nm})$ & Energy consumed $\left(\mathrm{fJ}=10^{-15} \mathrm{~J}\right)$ \\
\hline $\mathrm{Au}$ & 1.25 & 0.15 & 2.4 \\
$\mathrm{TDT} / \mathrm{Au}$ & 0.82 & 0.22 & 0.5 \\
$\mathrm{HDT} / \mathrm{Au}$ & 0.89 & 0.17 & 1.3 \\
$\mathrm{BPDT} / \mathrm{Au}$ & 1.40 & 0.30 & 2.8 \\
\hline $\mathrm{Ag}$ & 1.20 & 0.25 & 3.0 \\
TDT/Ag & 1.40 & 0.29 & 4.0 \\
HDT/Ag & 1.20 & 0.21 & 2.8 \\
\hline
\end{tabular}

Load-displacement (P-h) curves of samples on Au substrate are presented in Figure 1. Nanoindentation of the TDT/Au and HDT/Au samples (maximum depths of 0.82 and $0.89 \mathrm{~nm}$, resp.) show a stiffening response of about $30 \%$ in comparison to bare $\mathrm{Au}$ (maximum depth of $1.25 \mathrm{~nm}$ ), whereas no noticeable change, within the noise floor of the apparatus, in the maximum indentation depth is observed for the BPDT/Au (maximum depth of $1.40 \mathrm{~nm}$ ). On the other hand, the P-h curve for BPDT/Au shows clearly more hysteresis between loading and unloading than the data of bare $\mathrm{Au}$, whereas the hysteresis decreases by $\sim 80 \%$ and $\sim 46 \%$ in the presence of TDT and HDT SAMs, respectively (see the calculated consumed energy values of Table 1). It is well known that BPDT molecules form less ordered SAMs than alkanethiols and exhibit a higher density of gauche defects $[22,23]$, which allow a deeper penetration towards the Au substrate. On the other hand, while BPDT SAMs are chemically reactive, alkanethiols that are terminated by the inert $\mathrm{CH}_{3}$ group produce a lower surface energy, and due to their high hydrophobicity, they usually exhibit lower adhesion and friction [24]. Therefore, the pronounced hysteresis (higher consumed energy) in the P-h curve of the BPDT SAM on Au is mainly attributed to the lower degree of order and stronger interaction between the SAM molecules and the indenter tip than in the case of the hydrophobic $\mathrm{CH}_{3}-$ terminated alkanethiols and bare Au surface. Additionally, there may be some variations in the surface coverage of the molecules and in the tilt angle which depends on the chain length of the SAM molecule [25]. The angular degrees of freedom for a self-assembled monolayer of alkanethiol can be described by the tilt angle $\theta$ and the tilt direction $\chi$, as schematically represented in Figure 2. Based on a molecular dynamics study, Vemparala et al. [26] have reported that at room temperature the effect of chain length on the tilt angle is most pronounced. There is a jump of 3 and 6 degrees in both vertical and rotational alignments, respectively, as chain length increases from 14 to 16 [24, 25]. The resulting variation in the rotational alignment $\chi$ of the HDT molecules induces more molecular disorder explicating the observed higher hysteresis of the longer HDT molecules compared with the shorter TDT molecules (Figure 1).

To further study the features of the P-h curves, we performed nanoindentation experiments on TDT and HDT SAM covered Ag surfaces. In Figure 3 the mechanical responses of bare Ag and TDT and HDT coated Ag substrates are plotted. The TDT covered Ag sample exhibits a slightly

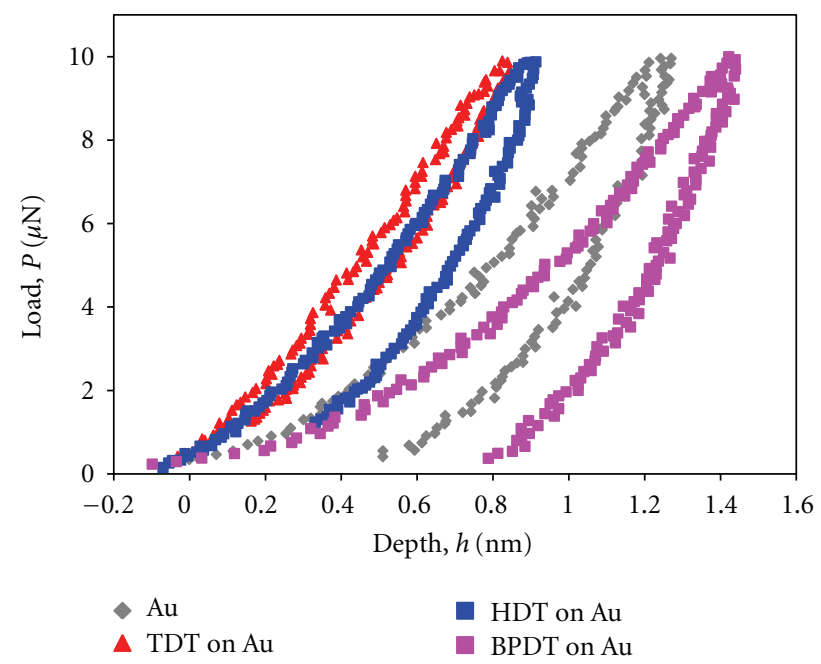

FIGURE 1: Averaged data obtained from nanoindentation of bare $\mathrm{Au}$ and TDT, HDT and BPDT SAMs on Au substrate.

deeper penetration depth $(1.40 \mathrm{~nm})$ and higher hysteresis (consumed energy $4 \mathrm{fJ}$ ) compared to bare Ag and the HDT covered Ag substrates (both have a penetration depth of $1.20 \mathrm{~nm}$ and consumed energies of 3.0 and $2.8 \mathrm{fJ}$, resp.). Both TDT/Ag, and HDT/Ag samples exhibit higher hysteresis, greater maximum indentation depths and larger creeping during the dwell time than the samples with the same SAMs on Au (Figure 1).

Monolayers grown on Au have tilted contact angles in the range of $20-40^{\circ}$ [24-29], while the ones on $\mathrm{Ag}$ tend to grow more perpendicular to the substrate surface with a tilt angle of approximately $6-7^{\circ}[16,26]$. In the case of Ag surfaces, the nearly perpendicular orientation of the molecules may initiate higher disorder already at the beginning of the indentation because there is no local preferential bending. Therefore, the noted large variations in the adhesive and elastic responses are in accordance with the expectations. When comparing the indentation curves obtained for bare $\mathrm{Au}$ and $\mathrm{Ag}$, they show similar indentation depths and hysteresis, Ag though exhibiting larger creeping during the dwell time than Au.

To account for the nanoindentation results, the P-h curve features are shown to be associated with several factors. The molecule chain length: the longer molecules show higher hysteresis compared with that of the shorter molecules. The terminal group of the molecule: a reactive group results 


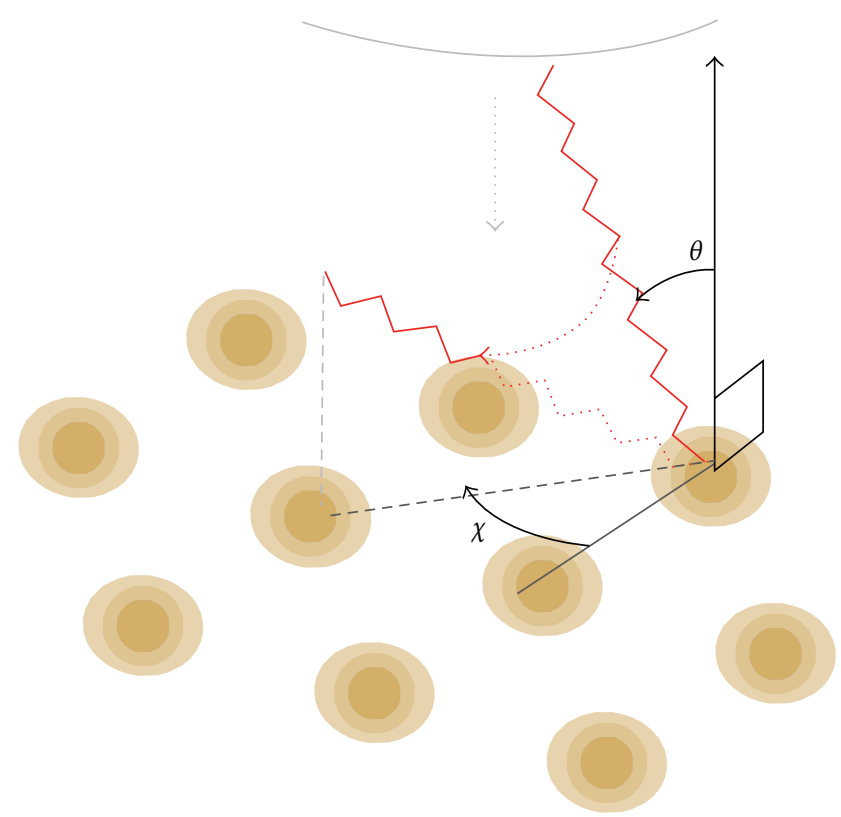

FIgURE 2: Schematics of the angular degrees of freedom of alkanethiol SAMs. The brown areas represent $S$ atoms of the molecules on the surface. Angle $\theta$ refers to the tilt of molecular axis with respect to substrate surface normal and $\chi$ defines the tilt direction, that is, it is derived from projection of molecule in the substrate plane.

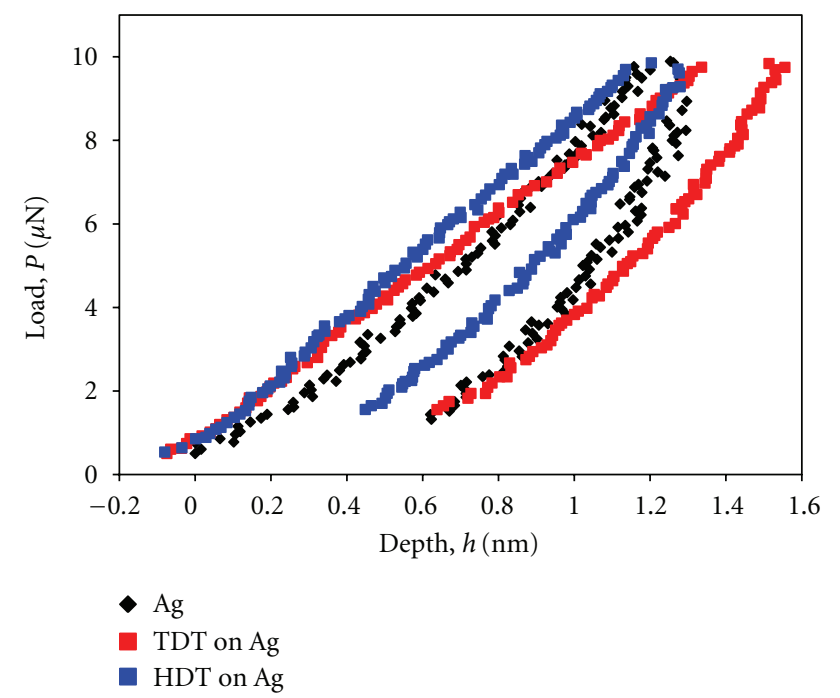

Figure 3: Averaged data obtained from nanoindentation of bare Ag, TDT and HDT SAMs on Ag substrate.

in pronounced hysteresis. The less ordered SAMs show deeper penetration depths. The substrate (Au versus $\mathrm{Ag}$ ) effect is expressed through the molecule tilt angle which it affects, substrate topography and through the varying surface coverage.

\section{Conclusions}

Mechanical responses of different thiolate monolayers (BPDT, TDT and HDT) on gold and silver substrates were studied experimentally with classical nanoindentation complemented with appropriate statistical analysis. A conical diamond tip with a $2 \mu \mathrm{m}$ tip radius was used in order to sample a larger number of SAM molecules and mitigating the impact of local effects. As indicated by the tip displacements in the P-h curves, the BPDT covered Au substrate was softer in mechanical response compared with the TDT and HDT SAMs, due to the lower degree of order of the BPDT molecules and stronger interaction between the SAM molecules and the indenter tip. On the other hand, more gauche defects and extended dislocations arise upon indentation into SAM coated Ag surfaces as a consequence of the SAM orientation. The herein proposed procedure enables a fast and efficient way of finding the essential mechanical response of monolayer structures needed for many practical applications.

\section{Acknowledgments}

The authors are grateful to Mr Mikko Heikkilä for the XRD measurements. The University of Helsinki, the Academy of Finland (Project nos. 121567, 134406, and 251531) as well as the Baltech scholarship are acknowledged for the financial support. This work has been partially supported by the Graduate School "Functional materials and processes" receiving funding from the European Social Fund under Project 1.2.0401.09-0079 in Estonia.

\section{References}

[1] T.-H. Fang, W.-Y. Chang, C.-I. Weng, and C.-N. Fang, "Surface and mechanical characteristics of alkanethiol self-assembled monolayers," Journal of Advanced Microscopy Research, vol. 6, no. 1, pp. 24-28, 2011.

[2] F. W. Delrio, C. Jaye, D. A. Fischer, and R. F. Cook, "Elastic and adhesive properties of alkanethiol self-assembled monolayers on gold," Applied Physics Letters, vol. 94, no. 13, Article ID 131909, 2009.

[3] B. Bhushan, "Nanotribology and nanomechanics in nano/biotechnology," Philosophical Transactions of the Royal Society A, vol. 366, no. 1870, pp. 1499-1537, 2008.

[4] C. W. Chang and J. D. Liao, "Nano-indentation at the surface contact level: applying a harmonic frequency for measuring contact stiffness of self-assembled monolayers adsorbed on Au," Nanotechnology, vol. 19, no. 31, Article ID 315703, 2008.

[5] T.-H. Fang, W.-J. Chang, Y.-C. Fan, and C.-I. Weng, "Molecular dynamics of contact behavior of self-assembled monolayers on gold using nanoindentation," Applied Surface Science, vol. 255, no. 21, pp. 8931-8934, 2009.

[6] T.-H. Fang, W.-Y. Chang, S.-J. Lin, and C.-N. Fang, "Interface dynamics and mechanisms of nanoindented alkanethiol selfassembled monolayers using molecular simulations," Journal of Colloid and Interface Science, vol. 345, no. 1, pp. 19-26, 2010.

[7] J. E. Houston and H. I. Kim, "Adhesion, friction, and mechanical properties of functionalized alkanethiol self-assembled monolayers," Accounts of Chemical Research, vol. 35, no. 7, pp. 547-553, 2002.

[8] M. I. Aponte, Effect of alkanethiol self-assembled monolayers on the plastic and elastic deformation of gold (111) films [Ph.D. thesis], Rutgers, The State University of New Jersey, 2010. 
[9] N. Satyanarayana, Tribology of organic self-assembled monolayers (SAMs) and thin-films on Si surface [Ph.D. thesis], National University of Singapore, 2007.

[10] M. Wang, Nanoindentation of thin organic films and selfassembled monolayers [Ph.D. thesis], The University of Texas at Austin, 2004.

[11] H. Liu and B. Bhushan, "Investigation of nanotribological properties of self-assembled monolayers with alkyl and biphenyl spacer chains (Invited)," Ultramicroscopy, vol. 91, no. 1-4, pp. 185-202, 2002.

[12] W.-Y. Chang, T.-H. Fang, and C.-N. Fang, "Molecular dynamics on interface and nanoscratch mechanisms of alkanethiol self-assembled monolayers," Journal of Physical Chemistry B, vol. 113, no. 45, pp. 14994-15001, 2009.

[13] R. Nowak, D. Chrobak, S. Nagao et al., "An electric current spike linked to nanoscale plasticity," Nature Nanotechnology, vol. 4, no. 5, pp. 287-291, 2009.

[14] R. Nowak, F. Yoshida, D. Chrobak, K. J. Kurzydlowski, T. Takagi, and T. Sasaki, "Nanoindentation examination of crystalline solid surfaces," in Encyclopedia of Nanoscience and Nanotechnology, S. H. Nalwa, Ed., pp. 313-374, American Association, 2011.

[15] L. Costelle, P. Jalkanen, M. T. Räisänen, L. Lind, R. Nowak, and J. Räisänen, "Mechanical response of nanometer thick selfassembled monolayers on metallic substrates using classical nanoindentation," Journal of Applied Physics, vol. 110, no. 11, Article ID 114301, 4 pages, 2011.

[16] D. Chrobak, K. Nordlund, and R. Nowak, "Nondislocation origin of GaAs nanoindentation pop-in event," Physical Review Letters, vol. 98, no. 4, Article ID 045502, 2007.

[17] Software for Molecular Modeling, Spartan'02 for Windows, Wavefunction Inc., Irvine, Calif, USA, 2002.

[18] U. Weckenmann, S. Mittler, K. Naumann, and R. A. Fischer, "Ordered self-assembled monolayers of 4,4'-biphenyldithiol on polycrystalline silver: suppression of multilayer formation by addition of tri-n-butylphosphine," Langmuir, vol. 18, no. 14, pp. 5479-5486, 2002.

[19] C. P. Kubiak, R. P. Andres, T. Bein et al., "Single electron tunneling in molecular nanostructures of crystalline gold clusters attached by dithiols to $\mathrm{Au}$ [111]: direct I(V) measurements of individual surface attached gold clusters by STM," Mathematical and Physical Sciences C, vol. 499, pp. 498-501, 1997.

[20] J. C. Love, L. A. Estroff, J. K. Kriebel, R. G. Nuzzo, and G. M. Whitesides, "Self-assembled monolayers of thiolates on metals as a form of nanotechnology," Chemical Reviews, vol. 105, no. 4, pp. 1103-1169, 2005.

[21] A. Ulman, "Formation and structure of self-assembled monolayers," Chemical Reviews, vol. 96, no. 4, pp. 1533-1554, 1996.

[22] W. Azzam, B. I. Wehner, R. A. Fischer, A. Terfort, and C. Wöll, "Bonding and orientation in self-assembled monolayer of oligophenyldithiols on Au substrates," Langmuir, vol. 18, no. 21, pp. 7766-7769, 2002.

[23] V. Kriegisch and C. Lambert, "Self-assembled monolayers of chromophores on gold surfaces," Topics in Current Chemistry, vol. 258, pp. 257-313, 2005.

[24] F. Schreiber, "Structure and growth of self-assembling monolayers," Progress in Surface Science, vol. 65, no. 5-8, pp. 151256, 2000

[25] P. Fenter, A. Eberhardt, K. S. Liang, and P. Eisenberger, "Epitaxy and chainlength dependent strain in self-assembled monolayers," The Journal of Chemical Physics, vol. 106, no. 4, pp. 1600-1608, 1997.
[26] S. Vemparala, B. B. Karki, R. K. Kalia, A. Nakano, and P. Vashishta, "Large-scale molecular dynamics simulations of alkanethiol self-assembled monolayers," Journal of Chemical Physics, vol. 121, no. 9, pp. 4323-4330, 2004.

[27] F. Chesneau, J. Zhao, C. Shen, M. Buck, and M. Zharnikov, "Adsorption of long-chain alkanethiols on $\mathrm{Au}(111)$ : a look from the substrate by high resolution $\mathrm{x}$-ray photoelectron spectroscopy," The Journal of Physical Chemistry C, vol. 114, no. 15, pp. 7112-7119, 2010.

[28] C. Waring, P. A. J. Bagot, M. W. P. Bebbington et al., "How penetrable are thioalkyl self-assembled monolayers?" Journal of Physical Chemistry Letters, vol. 1, no. 13, pp. 1917-1921, 2010.

[29] R. Yamada and K. Uosaki, "In situ scanning tunneling microscopy observation of the self-assembly process of alkanethiols on gold(111) in solution," Langmuir, vol. 14, no. 4, pp. 855-861, 1998. 

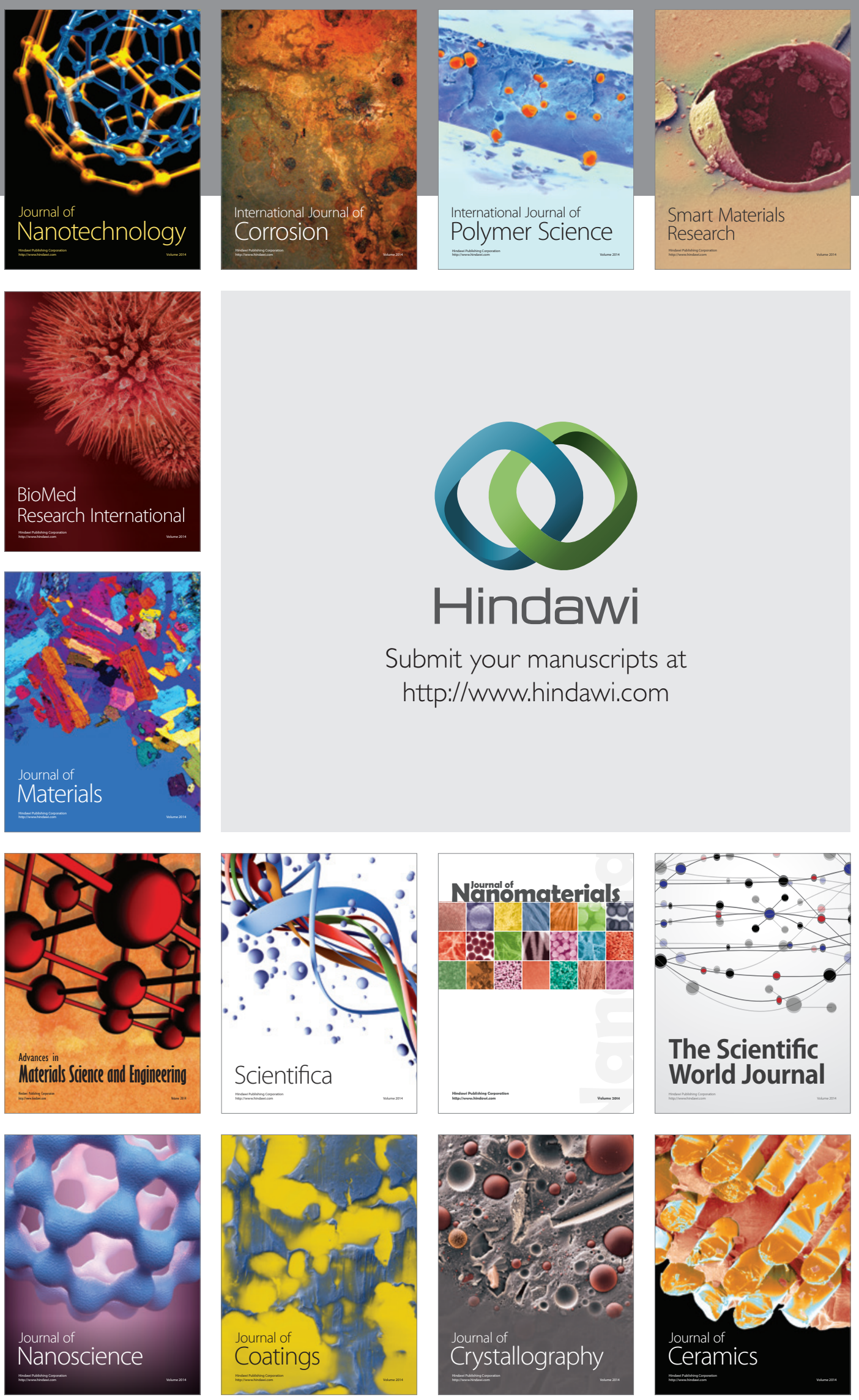

The Scientific World Journal

Submit your manuscripts at

http://www.hindawi.com

\section{World Journal}

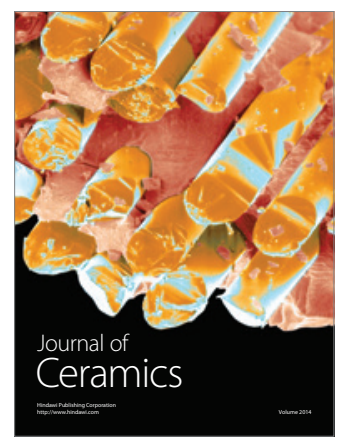

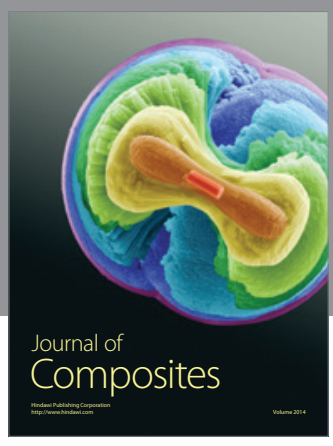
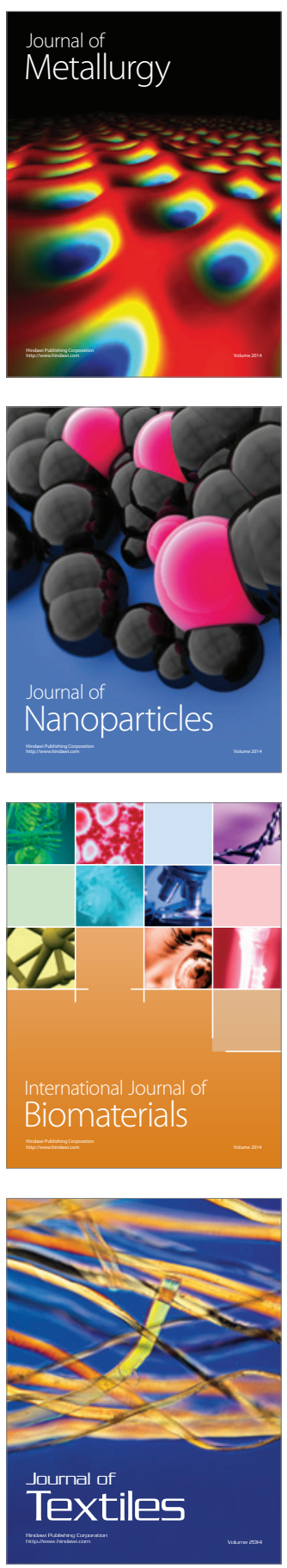